\title{
Ultrasonography to detect age-related changes in swallowing muscles
}

\author{
Takashi Mori $^{1,2}$ (D) Shinichi Izumi ${ }^{1} \cdot$ Yoshimi Suzukamo $^{1} \cdot$ Tatsuma Okazaki $^{1} \cdot$ Susumu Iketani ${ }^{2}$
}

Received: 19 March 2019 / Accepted: 19 July 2019 / Published online: 16 August 2019

(c) The Author(s) 2019

\section{Key summary points}

Aim To measure age-related changes in swallowing muscles by ultrasonography as a non-invasive method.

Findings By ultrasonography, we found that old people had a smaller geniohyoid muscle area and greater geniohyoid muscle brightness than young people. Our analyses indicated that age and whole-body skeletal muscle mass were associated with mass and quality of the geniohyoid muscle.

Message Ultrasonography effectively identified the association between atrophy of the swallowing muscles and aging and loss of whole-body skeletal muscle mass.

\begin{abstract}
Purpose Sarcopenia of swallowing muscles is a potential cause of dysphagia. We investigated age-related changes in mass and quality of swallowing muscles by ultrasonography as a non-invasive and convenient examination in subjects without dysphagia.

Methods A total of 104 subjects (34 males, 70 females) participated in this study. Age, physical status, and mass and strength of skeletal and swallowing muscles were investigated. Ultrasonography was performed to measure cross-sectional area and brightness of the geniohyoid muscle as a swallowing muscle. Calf circumference was measured to evaluate skeletal muscle mass. Hand grip strength was measured to evaluate skeletal muscle strength. Subjects were divided into two groups: young ( $<65$ years old) and old ( $\geq 65$ years old). We performed univariate and multivariate analyses to analyze the differences between the groups.

Results The number of subjects in the young group was 35, and 69 in the old group. The mean \pm SD of measurements in each group was as follows (young/old): age, $35.4 \pm 13.9 / 74.5 \pm 5.5$ years old; calf circumference, $37.4 \pm 4.1 / 33.9 \pm 2.7 \mathrm{~cm}$ $(p<0.001)$; hand grip strength, $35.6 \pm 10.2 / 25.8 \pm 7.6 \mathrm{~kg}(p<0.001)$; cross-sectional area of geniohyoid muscle, $229.5 \pm 52.2 / 174.1 \pm 40.7 \mathrm{~mm}^{2}(p<0.001)$; and brightness of geniohyoid muscle, $46.6 \pm 11.1 / 59.6 \pm 10.8(p<0.001)$. The old group had a significantly smaller geniohyoid muscle area and significantly greater geniohyoid muscle brightness than the young group $(p<0.01)$. Age and calf circumference were independent explanatory factors for geniohyoid muscle area $(p<0.01)$. Age and sex were independent explanatory factors for geniohyoid muscle brightness $(p<0.01)$.
\end{abstract}

Conclusions Ultrasonography revealed a smaller area and greater brightness, which suggested smaller mass and greater infiltration of fat, in the geniohyoid muscle in old people than in young people.

Keywords Swallowing $\cdot$ Muscle $\cdot$ Ultrasonography $\cdot$ Sarcopenia

Takashi Mori

tmori0117@yhaoo.co.jp

1 Department of Physical Medicine and Rehabilitation, Tohoku University Graduate School of Medicine, Sendai, Miyagi, Japan

2 Department of Oral and Maxillofacial Surgery, Dysphagia Rehabilitation Center, Southern Tohoku General Hospital, 7-115 Yatsuyamada, Koriyama, Fukushima 963-8563, Japan

\section{Background and purpose}

Sarcopenia increases physical dysfunction [1,2]. Previous research reported that sarcopenia might cause dysphagia [3-7]. Sarcopenia and dysphagia are important problems among old people; particularly, the prevalence of sarcopenic dysphagia was reported to be $32 \%$ in rehabilitation patients in Japan, a rapidly aging society [8]. Sarcopenic dysphagia is 
defined as a swallowing disorder due to sarcopenia of wholebody skeletal and swallowing muscles [3, 9].

As sarcopenia can be treated [1,2], confirming the status of muscles is important to prevent muscle dysfunction. Feng et al. and Molfenter et al. reported that old people had lower geniohyoid and pharyngeal muscle mass than young people when evaluated by CT or MRI, respectively $[10,11]$. Compared with CT and MRI, ultrasonography is characterized by being radiation-free, portable, easy to use, and having a low cost, and short examination time, thus, making it useful for screening many people in order to confirm their muscle status. Several reports evaluated swallowing muscle mass and brightness, later as a measure of muscle quality, by ultrasonography [12-19]. Muscle brightness suggests infiltration of fat [20]. However, age-related changes of these parameters measured by ultrasonography are unclear.

In this study, we measured age-related changes in swallowing muscle mass and quality by ultrasonography. We investigated the cross-sectional area and brightness of the geniohyoid muscle as a representative swallowing muscle and compared between young ( $<65$ years old) and old ( $\geq 65$ years old) people.

\section{Methods}

\section{Participants and parameters}

The study design was cross-sectional. Subjects were divided into two groups: young ( $<65$ years old) and old ( $\geq 65$ years old). Old subjects were recruited from three preventative care services and one regional preventative care event for older citizens, and young subjects were recruited from a medical facility. Inclusion criterions were independently living subjects without severe illness that affected their physical function. Subjects with dysphagia were excluded using Food intake LEVEL scale [21]. The study period was between Dec. 2017 and Feb. 2019. The ethical committees of Southern Tohoku General Hospital and Tohoku University approved this study. All patients were given documents and provided written informed consent prior to enrollment.

We assessed parameters concerning both skeletal and swallowing muscles. Age, sex, height, body weight, body mass index (BMI), calf circumference, hand grip strength, and Mini Nutritional Assessment ${ }^{\circledR}$ short form (MNA-SF) [22] score for nutritional status were assessed. The thickest part of the lower leg was assessed using a measure as a calf circumference. Calf circumference was regarded as an indicator of whole-body skeletal muscle mass [23]. The hand grip strength was measured using a Smedley hand dynamometer in the standing position. Hand grip strength was used as an indicator of whole-body muscle strength [1, 2]. The maximum strength was taken as a result. MNA-SF was used because nutritional status and dysphagia had a clear association [24]; it is a screening questionnaire to assess nutritional status consisting of six questions. A total score of $12-14$ points indicates a healthy condition, $8-11$ means risk, and 0-7 malnutrition.

For swallowing muscles, Food Intake LEVEL Scale (FILS) was measured to evaluate swallowing function. Maximum tongue pressure was measured to evaluate swallowing muscle strength. And, ultrasonography was performed. FILS is a validated ranking scale for evaluating swallowing function [20]. A score of 10 indicates normal function, 7-9 indicates mild dysphagia, 4-6 indicates moderate dysphagia and 1-3 indicates severe dysphagia. In this study, we considered subjects with a FILS score of 10 to have normal swallowing function, and those with FILS score 9 and under, to have dysphagia. Maximum tongue pressure was assumed to demonstrate swallowing muscle strength [25]. A tongue pressure measurement instrument (JMS, Hiroshima, Japan) was used to measure tongue pressure [26]. Subjects were instructed to press a balloon to their palate folds with their tongues. Tongue pressure measurements were performed three times for each subject, and the maximum score was taken as a result. The cross-sectional area of the geniohyoid muscle was used as an indicator of swallowing muscle mass. Brightness of the geniohyoid muscle was used as an indicator of swallowing muscle quality because high intensity in the muscle indicates the presence of fat [20].

\section{Ultrasonography}

We used a LOGIQ e V2 (GE Health Care Japan, Tokyo, Japan) and convex-array probe (Model No. is 4C-RS) for ultrasonography. We created a parameter setting button on the screen of the ultrasound machine for this study, labeled as "swallow" mode. In the swallow mode, the parameters were set as follows: gain, 60; dynamic range, 78; frequency, $4.0 \mathrm{MHz}$; depth, $7 \mathrm{~cm}$. Before every measurement, the tester set the ultrasound machine to "swallow" mode. The parameters were recorded with each ultrasound image simultaneously, and we checked the status of parameters in the image analyses. Subjects were instructed to sit on a chair, face forward, keep a neutral position, and close the mouth. We instructed the subjects not to move or swallow while the US pictures were being taken. A probe was set under the jaw in the central sagittal plane. Ultrasonography measurements were performed three times, and the mean score was taken as a result. The area and brightness of the geniohyoid muscles in the sagittal plane were calculated using image J (Fig. 1, all images belong to author T.M.). An area of interest was set in the manual polygon mode. Brightness was assessed by the mean echo level of the area of interest, and the range was $0-255$. Ultrasonography and analysis were performed 
Fig. 1 Ultrasonography method. a Posture during ultrasonography; b ultrasonography image of the geniohyoid muscle in the sagittal plane; $\mathbf{c}$ detection of an area of interest using image J. All images belong to author T.M.

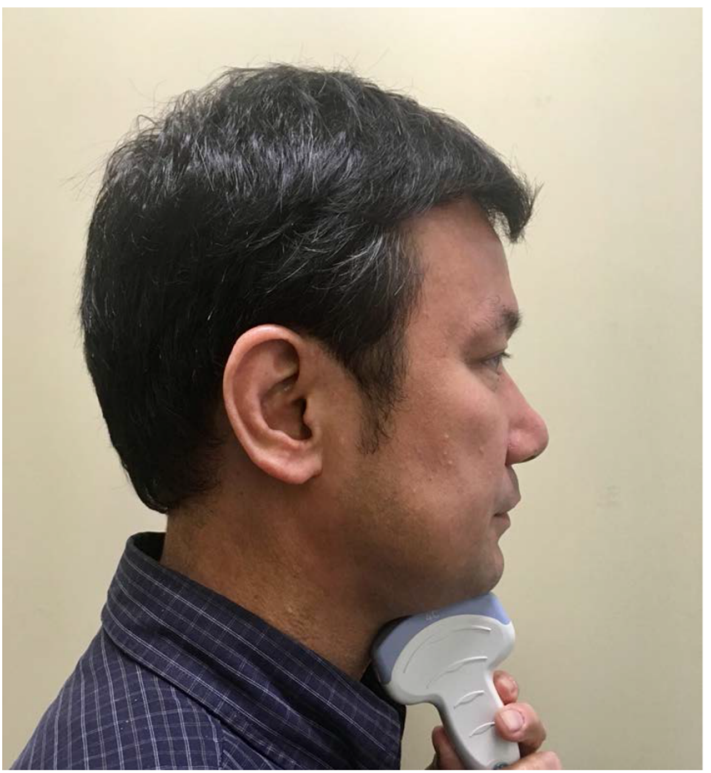

(a) posture in ultrasonography

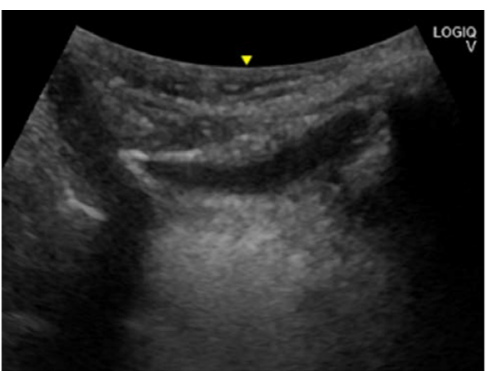

(b) geniohyoid muscle image in ultrasonography

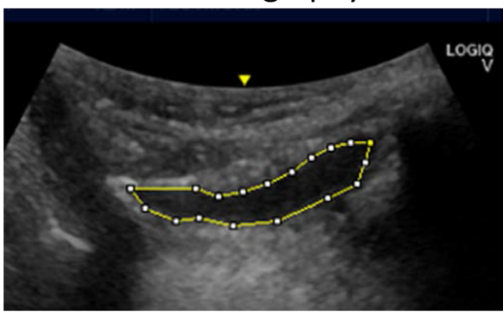

(c) area detection using image $\mathrm{j}$ by one speech therapist (T.M.) to reduce any potential bias caused by different testers.

\section{Statistical analysis}

The means of age, height, weight, BMI, calf circumference, hand grip strength, MNA-SF, maximum tongue pressure, and the cross-sectional area and brightness of the geniohyoid muscle were calculated. The median FILS scores were also calculated. We evaluated the relationships among the parameters using Pearson's product moment correlation coefficient. The subjects were divided into two groups: young ( $<65$ years old) and old ( $\geq 65$ years old). Univariate analyses were performed to investigate differences between the two groups using the Student's $t$ test. Conditions for sample size calculation were set as follows: $\alpha$ error, 0.05 ; power, 0.8 . The required sample size to detect differences in area and brightness of the geniohyoid muscle between the young and old groups was 11 in the young group and 22 in the old group. In multiple regression analysis, age, sex, height and calf circumference were chosen as explanatory variables for the area and brightness of the geniohyoid muscle because these parameters can affect skeletal muscle mass. All analyses were performed using EZR software [27, 28], $p$ values $<0.05$ were considered significant.

\section{Results}

A total of 104 subjects participated in this study, 34 males and 70 females. There were 35 subjects ( 18 males and 17 females) in the young group and 69 subjects (16 males and
53 females) in the old group. Table 1 shows significant differences between young and old groups in age, height, weight, calf circumference, hand grip strength, maximum tongue pressure, and geniohyoid muscle area and brightness. Table 2 shows differences between young and old groups in each gender. There were significant differences in area and brightness of the geniohyoid muscles between young and old subgroups of both the male and female groups. The old group had smaller area and greater brightness of the geniohyoid muscle (Figs. 2, 3). The area of the geniohyoid muscle was correlated with height, calf circumference, hand grip strength, and maximum tongue pressure, and was negatively correlated with age and brightness of the geniohyoid muscle (Table 3). The brightness of the geniohyoid muscle was correlated with age, and negatively correlated with height, calf circumference, hand grip strength and area of geniohyoid muscle (Table 3).

Tables 4 and 5 show the results of the multivariate regression models for the area and brightness of the geniohyoid muscle. Age and calf circumference were independent explanatory factors for a small geniohyoid muscle area. Age and sex were independent explanatory factors for great brightness of the geniohyoid muscle.

\section{Discussion}

In this study, the area of the geniohyoid muscle was associated with calf circumference, grip strength, and maximum tongue pressure. This muscle moves the hyoid bone and elevates the larynx in the swallowing motion [29]. Loss of geniohyoid muscle mass suggests atrophy of swallowing 
Table 1 Characteristics of the subjects

\begin{tabular}{lllll}
\hline & Total $(N=104)$ & Young $(N=35)$ & Old $(N=69)$ & $p$ \\
\hline Sex, male, $n(\%)$ & $70(67.3)$ & $18(51.4)$ & $16(23.2)$ & 0.007 \\
Age, years, mean (SD) & $61.3(20.5)$ & $35.4(13.9)$ & $74.5(5.5)$ & $<0.001$ \\
Height (cm), mean (SD) & $157.4(10.0)$ & $164.7(7.6)$ & $153.7(10.1)$ & $<0.001$ \\
Weight (kg), mean (SD) & $56.6(10.8)$ & $61.5(12.9)$ & $54.2(8.8)$ & 0.004 \\
BMI, mean (SD) & $22.7(3.1)$ & $22.5(3.6)$ & $22.9(2.9)$ & 0.647 \\
Calf circumference (cm), mean (SD) & $35.1(3.5)$ & $37.4(4.1)$ & $33.9(2.7)$ & $<0.001$ \\
Hand grip strength (kg), mean (SD) & $29.1(9.7)$ & $35.6(10.2)$ & $25.8(7.6)$ & $<0.001$ \\
MNA-SF, score, median (IQR) & $13(12-14)$ & $13(12-14)$ & $13(12-14)$ & $0.26^{*}$ \\
FILS, score, median (IQR) & $10(10-10)$ & $10(10-10)$ & $10(10-10)$ & n.s** \\
Maximum tongue pressure (kPa), mean (SD) & $37.1(7.9)$ & $41.2(7.7)$ & $35.1(7.3)$ & $<0.001$ \\
Geniohyoid muscle area (mm $\left.{ }^{2}\right)$, mean (SD) & $192.7(51.4)$ & $229.5(52.2)$ & $174.1(40.7)$ & $<0.001$ \\
Geniohyoid muscle brightness, mean (SD) & $55.2(12.3)$ & $46.6(11.1)$ & $59.6(10.8)$ & $<0.001$ \\
\hline
\end{tabular}

$p$ indicates the $p$ value of the Student's $t$ test except for MNA-SF and FILS

*Indicates the $p$ value of the Mann-Whitney $U$ test

**FILS scores for all the subjects were 10 points

Table 2 Comparison between young and old group in each gender

\begin{tabular}{|c|c|c|c|}
\hline & Young $(n=18)$ & Old $(n=16)$ & $p$ value \\
\hline \multicolumn{4}{|l|}{ (a) Male } \\
\hline Age, years, mean (SD) & $33.9(12.1)$ & $76.6(5.3)$ & $<0.001$ \\
\hline Height, cm, mean (SD) & $172.5(4.3)$ & $163.2(7.5)$ & $<0.001$ \\
\hline Weight, kg, mean (SD) & $70.8(8.0)$ & $63.1(8.3)$ & 0.009 \\
\hline BMI, mean (SD) & $23.9(3.2)$ & $23.6(2.1)$ & 0.796 \\
\hline Calf circumference, $\mathrm{cm}$, mean (SD) & $40.0(3.4)$ & $35.2(1.8)$ & $<0.001$ \\
\hline Hand grip strength, kg, mean (SD) & $44.1(5.8)$ & $34.6(8.7)$ & 0.001 \\
\hline MNA-SF, score, median (IQR) & $14(13-14)$ & $12.5(12-14)$ & 0.016 \\
\hline FILS, score, median (IQR) & $10(10-10)$ & $10(10-10)$ & n.s** \\
\hline Maximum tongue pressure, $\mathrm{kPa}$, mean (SD) & $43.4(9.2)$ & $33.7(6.3)$ & 0.001 \\
\hline Geniohyoid muscle area, $\mathrm{mm}^{2}$, mean (SD) & $262.8(42.1)$ & $190.7(49.1)$ & $<0.001$ \\
\hline \multirow[t]{2}{*}{ Geniohyoid muscle brightness, mean (SD) } & $40.6(8.7)$ & $54.2(8.6)$ & $<0.001$ \\
\hline & Young $(n=17)$ & Old $(n=53)$ & $p$ value \\
\hline \multicolumn{4}{|l|}{ (b) Female } \\
\hline Age, years, mean (SD) & $36.9(15.8)$ & $73.8(5.5)$ & $<0.001$ \\
\hline Height, cm, mean (SD) & $156.4(7.6)$ & 150.(4.8) & 0.001 \\
\hline Weight, kg, mean (SD) & $51.7(9.7)$ & $51.5(7.1)$ & 0.938 \\
\hline BMI, mean (SD) & $21.1(3.7)$ & $22.7(3.1)$ & 0.1 \\
\hline Calf circumference, $\mathrm{cm}$, mean (SD) & $34.5(2.7)$ & $33.5(2.8)$ & 0.189 \\
\hline Hand grip strength, kg, mean (SD) & $26.7(4.4)$ & $23.1(4.8)$ & 0.009 \\
\hline MNA-SF, score, median (IQR) & $38.9(5.0)$ & $35.5(7.5)$ & 0.091 \\
\hline FILS, score, median (IQR) & $10(10-10)$ & $10(10-10)$ & n.s** \\
\hline Maximum tongue pressure, $\mathrm{kPa}$, mean (SD) & $12(12-13)$ & $13(12-14)$ & 0.259 \\
\hline Geniohyoid muscle area, $\mathrm{mm}^{2}$, mean (SD) & $194.2(36.8)$ & $169.0(36.9)$ & 0.017 \\
\hline Geniohyoid muscle brightness, mean (SD) & $53.0(10.0)$ & $61.2(10.9)$ & 0.007 \\
\hline
\end{tabular}

$p$ indicates the $p$ value of the Student's $t$ test except for MNA-SF and FILS

*Indicates the $p$ value of the Mann-Whitney $U$ test

**FILS scores for all the subjects were 10 points 


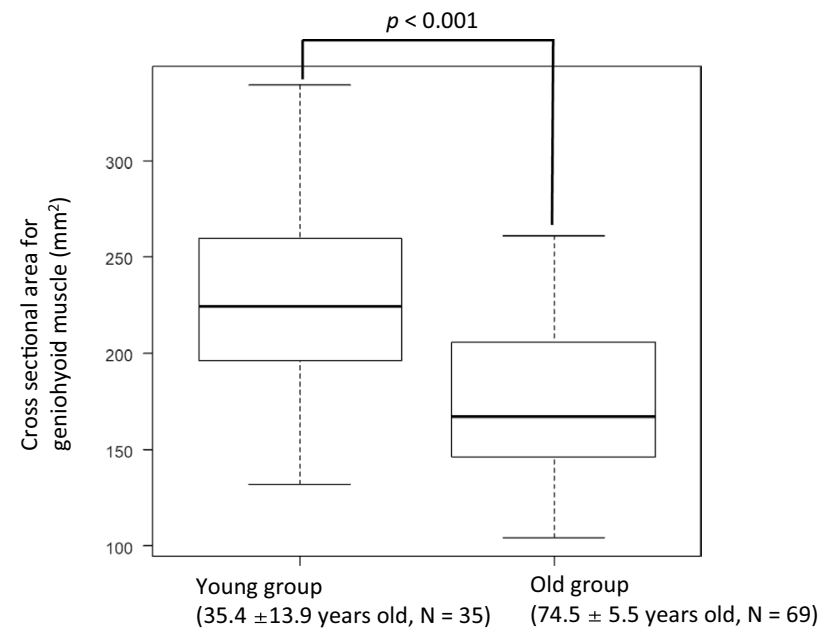

Fig. 2 Cross-sectional area of the geniohyoid muscle in the young and old groups. $p$ value by Student's $t$ test

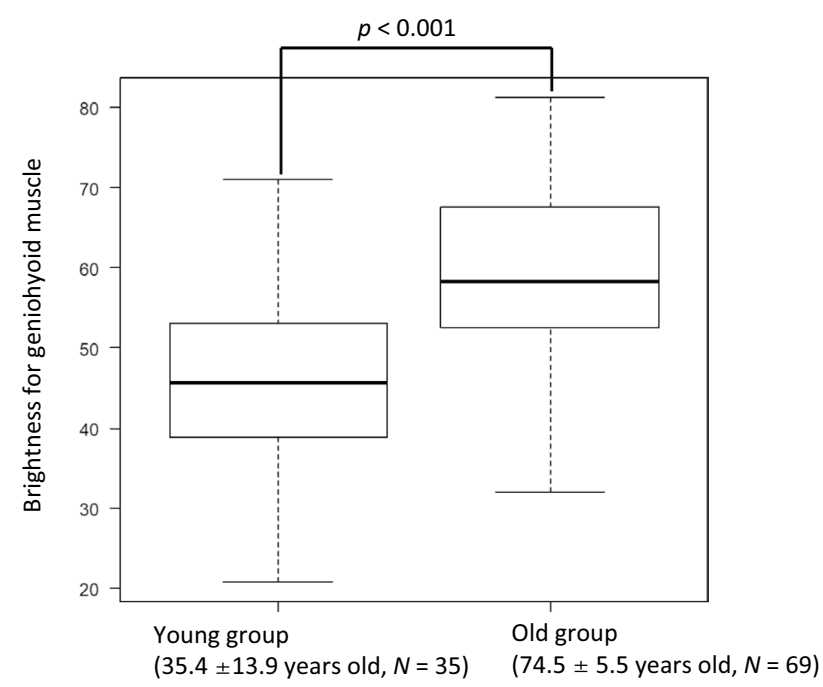

Fig. 3 Brightness of the geniohyoid muscle in the young and old groups. $p$ value by Student's $t$ test

muscles. Calf circumference is an indicator of whole-body skeletal muscle mass [22] and grip strength is an indicator of whole-body skeletal muscle strength $[1,2]$. Maximum tongue pressure is an indicator of swallowing muscle strength [24]. In the univariate analysis, the area of the geniohyoid muscle was correlated with calf circumference $(r=0.58, p<0.01)$, hand grip strength $(r=0.68, p<0.01)$, and maximum tongue pressure $(r=0.39, p<0.01)$. These results indicated that the area of the geniohyoid muscle, as a representative of swallowing muscle mass, was associated with whole-body skeletal muscle mass, and whole-body and swallowing muscle strength. These results are consistent with those of previous research. Yoshimi et al. reported that geniohyoid muscle area in the coronal plane correlated with trunk muscle mass index and tongue pressure [30]. Tamura et al. reported that tongue thickness in the coronal plane correlated with arm muscle area [14]. Baba et al. reported that geniohyoid muscle area in the coronal plane correlated with maximum tongue pressure [17].

Our data suggested that the area of the swallowing muscle decreased with aging. Old people had a smaller geniohyoid muscle area and greater brightness than young people. The sample size to detect a significant difference between the two groups in this study was sufficient (calculated size was 33 , and the actual size was 104). These results indicated that swallowing muscle mass and quality decreased with age and whole-body muscle mass. In the multivariate analyses, age $(p<0.01)$ and calf circumference $(p<0.01)$ were independent explanatory factors for loss of geniohyoid muscle mass. These results indicated that swallowing muscle mass was affected by age and whole-body skeletal muscle mass. Age $(p<0.01)$ and sex $(p<0.01)$ were independent risk factors for the low quality of the geniohyoid muscles. These results are consistent with those of previous research. Feng et al. used CT and reported that old people had a smaller geniohyoid muscle area in the sagittal plane than young people [10]. Molfenter et al. used MRI and reported that old people had a smaller pharyngeal muscle area in the horizonal plane than young people [11]. Baba et al. used ultrasonography and reported that geniohyoid muscle area in the coronal plane correlated with age [17]. As far as we know, there has been no previous research showing age-related changes in brightness of the geniohyoid muscle by ultrasonography, suggesting infiltration of fat into the muscle.

Our study showed that ultrasonography can measure the differences in the geniohyoid muscle area in the sagittal plane between young and old people and is the first to demonstrate the differences in geniohyoid muscle brightness between young and old people.

Sarcopenia can be treated by resistance training and nutrition. [1, 2]. Feng et al. and Baba et al. reported a correlation between swallowing function and swallowing muscle mass [10,17]. If sarcopenia of the swallowing muscles is detected at an early stage, swallowing muscle training should be started to prevent sarcopenic dysphagia.

There are two limitations in this study. First, muscle area measurement by ultrasonography was reported to be correlated with MRI measurement [31, 32]. However, for geniohyoid muscle area, an association between ultrasonography and MRI is not clear. In this study, we did not examine the association between ultrasonography and MRI for the measurement of geniohyoid muscle area. Second, effects of thoracic kyphosis or cervical lordosis on US measurements were not evaluated. To avoid stretching the geniohyoid muscle, neck position of the subjects was adjusted to central position. However, the presence of thoracic kyphosis or cervical lordosis was not assessed. 
Table 3 Univariate correlations

\begin{tabular}{lccccccccc}
\hline & Age & Height & Weight & BMI & CC & HG & MTP & GH area & GH br. \\
\hline Age & 1 & $-0.54^{*}$ & -0.06 & 0.02 & $-0.49^{*}$ & $-0.49^{*}$ & $-0.39^{*}$ & $-0.58^{*}$ & $0.52^{*}$ \\
Height & & 1 & -0.03 & -0.18 & $0.56^{*}$ & $0.76^{*}$ & $0.24^{*}$ & $0.59^{*}$ & $-0.50^{*}$ \\
Weight & & & 1 & $0.987^{*}$ & 0.15 & 0.05 & 0.12 & 0.04 & 0.03 \\
BMI & & & & 1 & 0.04 & -0.07 & 0.07 & -0.06 & 0.10 \\
CC & & & & & 1 & $0.58^{*}$ & $0.43^{*}$ & $0.58^{*}$ & $-0.30^{*}$ \\
HG & & & & & & 1 & $0.33^{*}$ & $0.68^{*}$ & $-0.51^{*}$ \\
MTP & & & & & & 1 & $0.39^{*}$ & -0.14 \\
GH area & & & & & & & & 1 & $-0.47^{*}$ \\
GH br. & & & & & & & & & 1 \\
\hline
\end{tabular}

Results show Pearson's correlation coefficients, *indicates the $p$ value $<0.05$

$C C$ calf circumference, $H G$ hand grip strength, $M T P$ maximum tongue pressure, $G H$ area, cross-sectional area of the geniohyoid muscle in the sagittal plane, $G H \mathrm{br}$. brightness of the geniohyoid muscle in the sagittal plane

Table 4 Multivariate regression analysis for area of the geniohyoid muscle

\begin{tabular}{lllllr}
\hline & $\begin{array}{l}\text { Estimated regres- } \\
\text { sion coefficient }\end{array}$ & $\begin{array}{l}\text { Lower 95\% confi- } \\
\text { dence interval }\end{array}$ & $\begin{array}{l}\text { Upper 95\% confi- } \\
\text { dence interval }\end{array}$ & Standard error & $p$ value \\
\hline Intercept) & 26.58 & -228.71 & 281.86 & 128.66 & 0.84 \\
Age & -0.87 & -1.33 & -0.40 & 0.23 & $<0.01$ \\
Sex & -19.22 & -44.72 & 6.27 & 12.84 & 0.14 \\
Height & 0.69 & -0.65 & 2.03 & 0.68 & 0.31 \\
CC & -29 & -46.586 & -10.492 & 9.097 & $<0.01$ \\
\hline
\end{tabular}

Adjusted $R^{2}=0.48, p$ value $<0.001$

$C C$ Calf circumference

Table 5 Multivariate regression analysis for brightness of the geniohyoid muscle

\begin{tabular}{lcccrr}
\hline & $\begin{array}{l}\text { Estimated regres- } \\
\text { sion coefficient }\end{array}$ & $\begin{array}{l}\text { Lower 95\% confi- } \\
\text { dence interval }\end{array}$ & $\begin{array}{l}\text { Upper 95\% confi- } \\
\text { dence interval }\end{array}$ & Standard error & $p$ value \\
\hline Intercept) & 7.74 & -59.52 & 75.00 & 33.90 & 0.82 \\
Age & 0.30 & 0.18 & 0.42 & 0.06 & $<0.01$ \\
Sex & 11.40 & 4.68 & 18.12 & 3.39 & $<0.01$ \\
Height & 0.00 & -0.35 & 0.36 & 0.18 & 0.99 \\
CC & 0.59 & -0.10 & 1.28 & 0.35 & 0.09 \\
\hline
\end{tabular}

Adjusted $R^{2}=0.39, p$ value $<0.001$

$C C$ Calf circumference
In conclusion, we suggest that atrophy and fatty change of the swallowing muscles are associated with aging and loss of whole-body skeletal muscles, and efficacy of the ultrasonography in their evaluation.

Acknowledgements We are grateful to Yumi Yamada, Tatsutya Bando, Yutaro Ohira, Yukari Ishikawa, Hidenori Oriuchi and Ippei Izumi for their support with data collection.

\section{Compliance with ethical standards}

Conflict of interest Takashi Mori received article processing charge from HEALTH TECHNOLOGY CO., LTD. Shinichi Izumi, Yoshimi Suzumkamo, Tatsuma Okazaki, and Susumu Iketani declare no conflicts of interest. 
Ethical approval Ethical committee of Southern Tohoku General Hospital and Tohoku University approved this study. All procedures performed in studies involving human participants were in accordance with the 1964 Helsinki declaration and its later amendments or comparable ethical standards.

Informed consent All patients were given documents and provided written informed consent prior to enrollment. Additional informed consent was obtained from all individual participants for whom identifying information is included in this article.

Open Access This article is distributed under the terms of the Creative Commons Attribution 4.0 International License (http://creativeco mmons.org/licenses/by/4.0/), which permits unrestricted use, distribution, and reproduction in any medium, provided you give appropriate credit to the original author(s) and the source, provide a link to the Creative Commons license, and indicate if changes were made.

\section{References}

1. Cruz-Jentoft AJ, Landi F, Schneider SM et al (2014) Prevalence of and interventions for sarcopenia in aging adults: a systematic review. Report of the International Sarcopenia Initiative (EWGSOP and IWGS). Age Aging 43(6):748-759. https://doi. org/10.1093/ageing/afu115

2. Cruz-Jentoft AJ, Bahat G, Bauer J et al (2019) Sarcopenia: revised European consensus on definition and diagnosis. Age Aging 48(1):16-31. https://doi.org/10.1093/ageing/afy169

3. Wakabayashi H (2014) Presbyphagia and sarcopenic dysphagia: association between aging, sarcopenia, and deglutition disorders. J Frailty Aging 3(2):97-103. https://doi.org/10.14283/jfa.2014.8

4. Wakabayashi H, Sakuma K (2014) Rehabilitation nutrition for sarcopenia with disability: a combination of both rehabilitation and nutrition care management. J Cachexia Sarcopenia Muscle 5(4):269-277. https://doi.org/10.1007/s13539-014-0162-x

5. Maeda K, Akagi J (2015) Decreased tongue pressure is associated with sarcopenia and sarcopenic dysphagia in the older. Dysphagia 30(1):80-87. https://doi.org/10.1007/s00455-014-9577-y

6. Maeda K, Takaki M, Akagi J (2017) Decreased skeletal muscle mass and risk factors of sarcopenic dysphagia: a prospective observational cohort study. Gerontol A Biol Sci Med Sci 27(9):1290-1294. https://doi.org/10.1093/gerona/glw190

7. Fujishima I, Fujiu-Kurachi M, Arai H et al (2019) Sarcopenia and dysphagia: position paper by four professional organizations. Geriatr Gerontol Int 19:91-97. https://doi.org/10.1111/ggi.13591

8. Wakabayashi H, Takahashi R, Murakami M (2019) The prevalence and prognosis of sarcopenic dysphagia in patients dysphagia in patients who require dysphagia rehabilitation. J Nutr Health Aging 23(1):84-88. https://doi.org/10.1007/s12603-018-1117-2

9. Mori T, I Fujishima, H Wakabayashi, et al. (2017). Development, reliability, and validity of a diagnostic algorithm for sarcopenic dysphagia. JCSM Clinical Reports 2 (2):1-10. https://jcsm-clini cal-reports.info/index.php/jcsm-cr/article/view/17/18. Accessed 1 Feb 2019

10. Feng X, Todd T, Lintzenich CR et al (2013) Aging-related geniohyoid muscle atrophy is related to aspiration status in healthy older adults. Gerontol A Biol Sci Med Sci 68(7):853-860. https ://doi.org/10.1093/gerona/gls225

11. Molfenter SM, Amin MR, Branski RC et al (2015) Age-related changes in pharyngeal lumen size: a retrospective MRI analysis. Dysphagia 30(3):321-327. https://doi.org/10.1007/s0045 5-015-9602-9
12. Watkin KL, Diouf I, Gallagher TM et al (2001) Ultrasonic quantification of geniohyoid cross-sectional area and tissue composition: a preliminary study of age and radiation effects. Head Neck 23(6):467-474. https://doi.org/10.1002/hed.1061

13. Feng X, Cartwright MS, Walker FO et al (2015) Ultrasonographic evaluation of geniohyoid muscle and hyoid bone during swallowing in young adults. Laryngoscope 125(8):1886-1891. https://doi. org/10.1002/lary.25224

14. Tamura F, Kikutani T, Tohara T et al (2012) Tongue thickness relates to nutritional status in the elderly. Dysphagia 27:556-561. https://doi.org/10.1007/s00455-012-9407-z

15. Shimizu S, Hanayama K, Nakato K et al (2016) Ultrasonographic evaluation of geniohyoid muscle mass in perioperative patients. Kawasaki Med J 42:47-56. https://doi.org/10.11482/ KMJ-E42(2)47

16. Shimizu S, Hanayama K, Metani H et al (2016) Retest reliability of ultrasonic geniohyoid muscle measurement. Jpn J Compr Rehabil Sci. https://doi.org/10.11336/jjcrs.7.55

17. Baba T, Goto T, Fujimoto K et al (2017) Age-related changes in geniohyoid muscle morphology predict reduced swallowing function. J Oral Health Biosci 30:18-25. https://doi.org/10.20738 /johb.30.1_18

18. Tamura F, Kikutani T, Furuya $\mathrm{H}$ et al (2017) A study of tongue thickness in older people in Japan. Med Res Arch 5:1-14. https:// doi.org/10.1007/s00455-012-9407-z

19. Ogawa N, Mori T, Fujishima I et al (2018) Ultrasonography to measure swallowing muscle mass and quality in older patients with sarcopenic dysphagia. JAMDA 19(6):516-522. https://doi. org/10.1016/j.jamda.2017.11.007

20. Reimers K, Reimers CD, Wagner Stefen et al (1993) Skeletal muscle sonography: a correlative study of echogenicity and morphology. J Ultrasound Med 12:73-77. https://doi.org/10.7863/ jum.1993.12.2.73

21. Kunieda K, Ohno T, Fujishima I et al (2013) Reliability and validity of a tool to measure the severity of dysphagia the food intake LEVEL scale. J Pai Symptom Management 46(2):201-206. https ://doi.org/10.1016/j.jpainsymman.2012.07.020

22. Kaiser M, Bauer J, Ramsch C et al (2009) Validation of the mini nutritional assessment short-form (MNA ${ }^{\circledR}-\mathrm{SF}$ ); a practical tool for identification of nutritional status. J Nutr Heal Aging 13:782-788. https://doi.org/10.1007/s12603-009-0214-7

23. Kawakami R, Murakami H, Sanada K et al (2015) Calf circumference as a surrogate marker of muscle mass for diagnosing sarcopenia in Japanese men and women. Geriatr Gerontol Int. 15:969-976. https://doi.org/10.1111/ggi.12377

24. Serra-Prat M, Palomera M, Gomez C et al (2012) Oropharyngeal dysphagia as a risk factor for malnutrition and lower respiratory tract infection in independently older persons: a population -based prospective study. Age Ageing 41(3):376-381. https://doi. org/10.1093/ageing/afs006

25. Steel MC (2013) Optimal approaches for measuring tongue-pressure functional reserve. J Aging Res 2013:542909. https://doi. org/10.1155/2013/542909

26. Utanohara Y, Hayashi R, Yoshikawa M et al (2008) Standard values of maximum tongue pressure taken using a newly developed disposable tongue pressure measurement device. Dysphagia 23:286-290. https://doi.org/10.1007/s00455-007-9142-z

27. Kanda Y (2013) Investigation of the freely available easy-touse software 'EZR' for medical statistics. Bone Marrow Transpl 48:452-458. https://doi.org/10.1038/bmt.2012.244

28. Institute for Statistics and Mathematics of Wirtschaftsuniversität Wien. The Comprehensive R Archive Network. (2019). https:// cran.r-project.org/. Accessed 22 Feb 2019

29. Palmer JB, Rudin NJ, Lara G et al (1992) Coordination of mastication and swallowing. Dysphagia 7:187-200 (PMID: 1308667) 
30. Abe T, Kondo M, Kawakami Y et al (1994) Prediction equations for body-composition of Japanese adults by B-Mode ultrasound. Am J Hum Biol 6:161-170. https://doi.org/10.1002/ajhb.13100 60204

31. Sanada K, Kearns CF, Midorikawa T et al (2006) Prediction and validation of total and regional skeletal muscle mass by ultrasound in Japanese adults. Eur J Appl Physiol 96:24-31. https:// doi.org/10.1007/s00421-005-0061-0

32. Yoshimi K, Hara K, Tohara H et al (2018) Relationship between swallowing muscles and trunk muscle mass in healthy elderly individuals: a cross-sectional study. Arch Gerontol Geriatr 79:2126. https://doi.org/10.1016/j.archger.2018.07.018

Publisher's Note Springer Nature remains neutral with regard to jurisdictional claims in published maps and institutional affiliations. 